\title{
Extrapleural pneumonectomy with en bloc myocardial resection for advanced thymoma
}

Fatima Iqbal, MD, ${ }^{\mathrm{a}}$ Christine Shokrzadeh, MD, ${ }^{\mathrm{b}}$ Ranjana Nawgiri, $\mathrm{MD},{ }^{\mathrm{a}}$ and Ikenna Okereke, $\mathrm{MD}^{\mathrm{c}}$

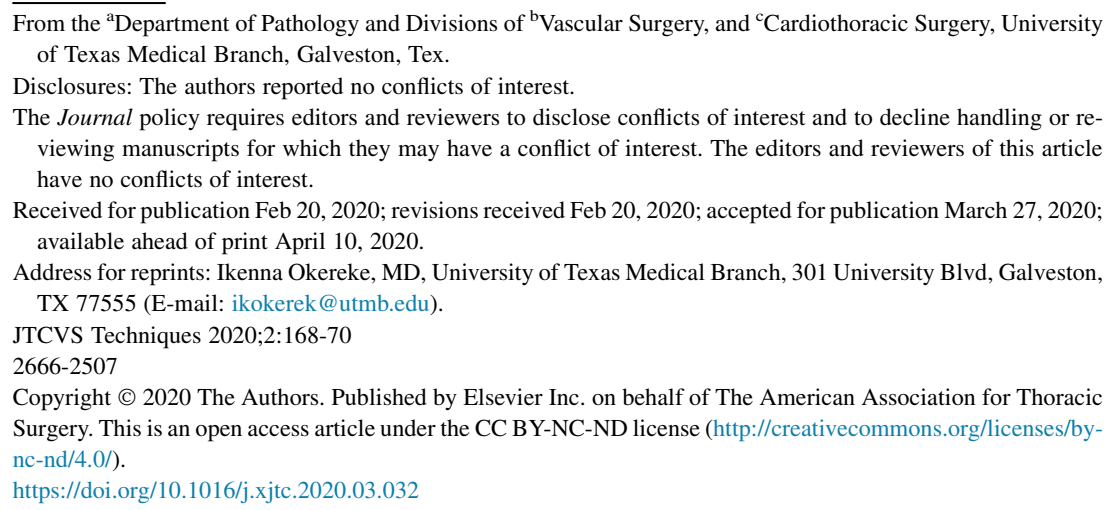

$\square$ Video clip is available online.

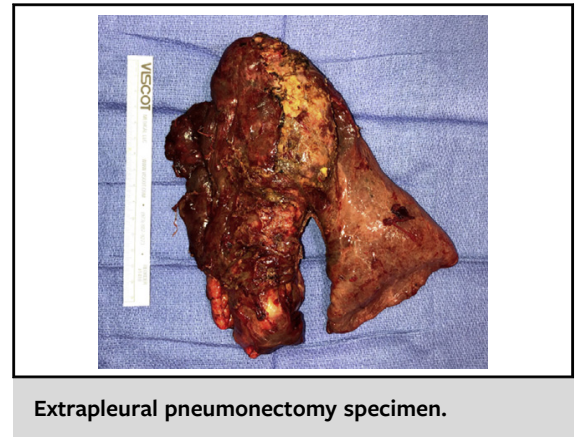

\begin{abstract}
CENTRAL MESSAGE
Some patients with thymoma

and myasthenia may not recover without surgery. Appropriate management can allow for successful resection.
\end{abstract}

See Commentaries on pages 171 and 173.
Treatment of patients with thymoma and myasthenia crisis can be challenging. After stabilization with medical management, surgical resection is recommended. ${ }^{1,2}$ Some patients will not significantly recover until the tumor is removed, however. We report a case of resolution of myasthenia crisis following extrapleural pneumonectomy in a young patient.

\section{CASE REPORT}

A 36-year-old woman had undergone a sternotomy, subtotal thymectomy, and debulking of an advanced thymoma lesion at an outside institution 9 years before presentation. The lesion was intimately associated with the myocardium of the left ventricle and was deemed unresectable. She received 2 cycles of carboplatin and Taxol treatment shortly after surgery but was lost to follow-up for several years.

She presented to our institution with shortness of breath and weakness. Serum studies were positive for acetylcholine receptor antibody. Computed tomography scan showed a large mediastinal mass with pleural and pericardial involvement (Figure 1). Cardiac magnetic resonance imaging showed the tumor to abut the left ventricle myocardium and left main pulmonary artery. There was no definite invasion into the ventricle, however. There was also no evidence of remaining thymus on the right side. The patient was given 3 cycles of chemotherapy with minimal tumor response.

She developed respiratory distress after the third cycle and was intubated. She was begun on intravenous immunoglobulin and plasmapheresis. After 2 weeks without significant improvement in respiratory status, a tracheostomy was performed. She recovered somewhat but was still unable to be weaned off of ventilator support.

After discussion with the multidisciplinary team, surgery was scheduled. Seventeen days after tracheostomy, she underwent a left thoracotomy, extrapleural pneumonectomy, and partial resection of the left ventricle (Figure 2). We felt that a left thoracotomy would avoid a redo sternotomy and that all right-sided thymus had likely been resected previously during the sternotomy surgery. Although preparations were made for cardiopulmonary bypass, a partialthickness myocardial resection was able to be performed without bypass (Video 1). A large portion of the pericardium was resected, and pericardial reconstruction was performed using a bovine pericardial patch. All gross tumor was resected. There was no diaphragmatic involvement. Estimated blood loss was $300 \mathrm{~mL}$.

Pathologic examination revealed a World Health Organization type B2, Masaoka IVa thymoma with negative 


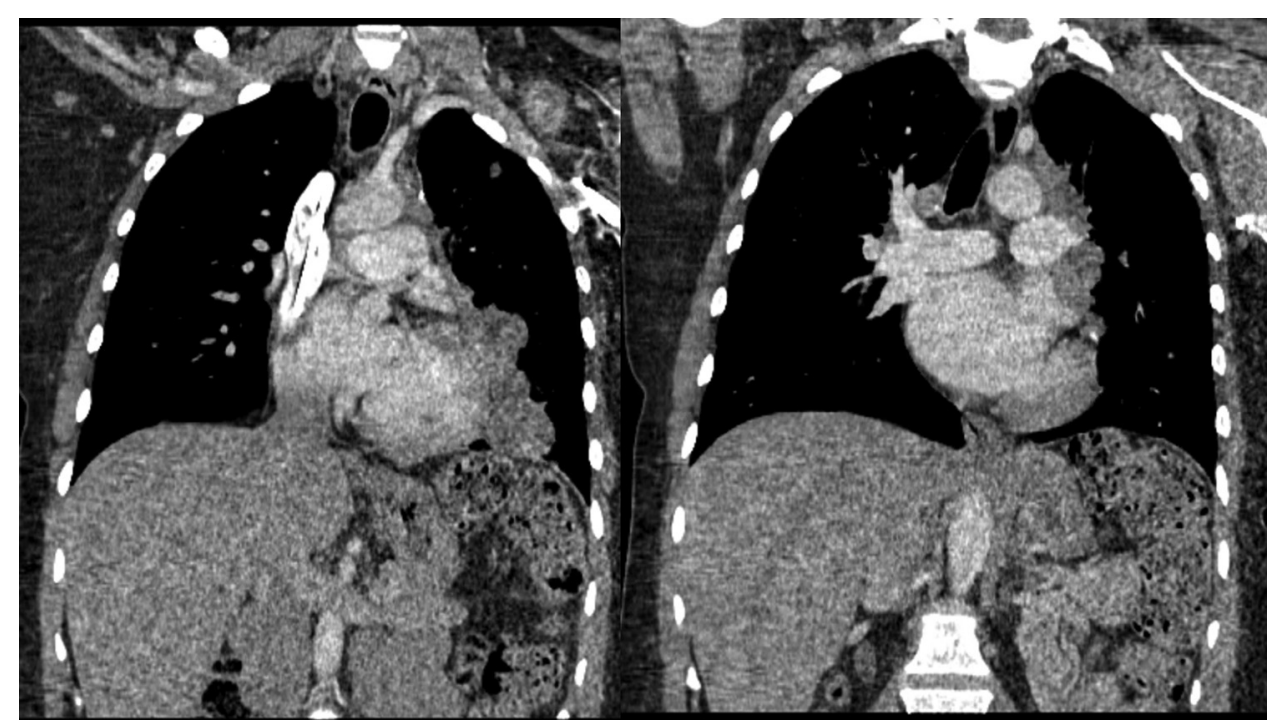

FIGURE 1. Computed tomography scan showing extensive thymoma mass with pleural and pericardial involvement.

margins. The thymoma mass measured $10 \times 6.5 \times 3 \mathrm{~cm}$. The patient experienced rapid improvement in her myasthenia symptoms. She was able to be weaned from the ventilator within 1 week of surgery. She was eventually discharged to a rehabilitation facility with only intermittent oxygen. She experienced 1 minor relapse of symptoms 3 months after surgery but was otherwise in good condition. On most recent follow-up 8 months after surgery she was free of symptoms and computed tomography scan showed no evidence of recurrent disease. The patient provided informed consent for the publication of the case.

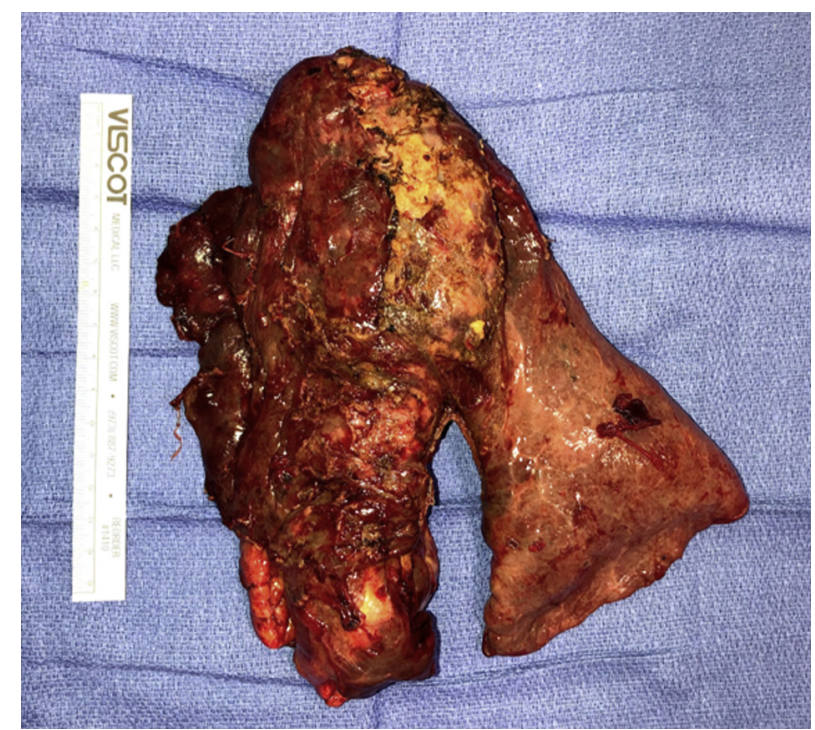

FIGURE 2. En bloc resection specimen including entire left lung, pericardium, and thymoma mass.

\section{DISCUSSION}

Surgical resection for patients with thymoma and myasthenia crisis is usually deferred until the patient has demonstrated significant improvement in respiratory symptoms, ${ }^{3}$ but many patients will not be able to achieve a demonstrable recovery without removing the source of the myasthenia gravis. Despite aggressive medical management for over 4 weeks, including intravenous immunoglobulin and plasmapheresis, our patient was not able to be weaned from the ventilator. Although pneumonectomy was associated with high risk, ${ }^{4,5}$ she was young and there did not appear to be alternative treatment strategies. A preoperative tracheostomy made her perioperative management much more straightforward, however, and complete resection was achieved. In retrospect, earlier tracheostomy may have decreased overall length of stay.

The timing of pneumonectomy was difficult, as we were hoping for successful weaning off the ventilator and

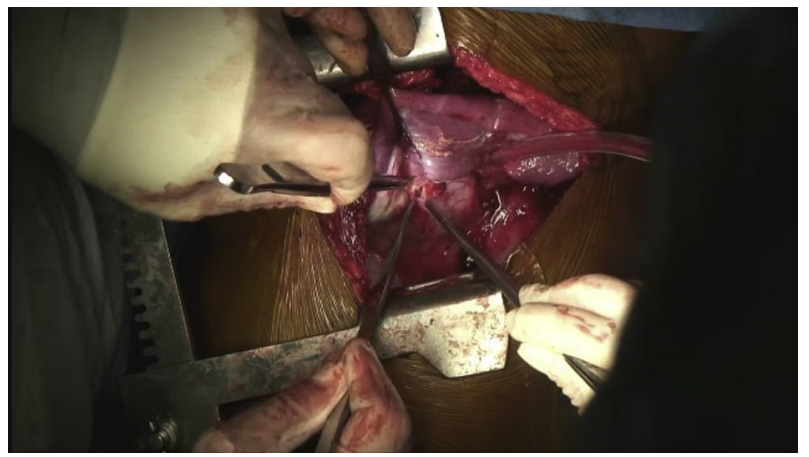

VIDEO 1. Video showing the dissection of the thymoma off of the left ventricle myocardium, division of the left main pulmonary artery, and removal of the specimen. Video available at: https://www.jtcvs.org/ article/S2666-2507(20)30185-1/fulltext. 
improvement in respiratory status as per traditional practice. After 4 weeks, however, we felt that her chances of recovery were low without removing the source of her symptoms by completely resecting the thymoma mass. We did not feel that waiting longer would lead to an improvement in respiratory function. Although she was ventilator-dependent at this point, she was able to communicate. After a thorough discussion, there was consensus multidisciplinary agreement to proceed with this very high-risk surgery and the patient adamantly wanted surgery.

Although our patient improved dramatically with surgery, it cannot be assumed that her myasthenia gravis has been completely cured. This fact is evidenced by her minor relapse months after resection.

We would recommend this aggressive approach only in very select patients. If our patient had been older or had any significant comorbidities, we would have likely not advised that she undergo surgery. However, in select patients with advanced thymoma and myasthenia crisis, extensive resections to remove all disease may be possible with appropriate planning.

\section{References}

1. Sanders DB, Wolfe GI, Benetar M, Evoli A, Gilhus NE, Illa I, et al. International consensus guidance for management of myasthenia gravis: executive summary. Neurology. 2016;87:419-25.

2. Kondo K, Monden Y. Thymoma and myasthenia gravis: a clinical study of 1,089 patients from Japan. Ann Thorac Surg. 2005;79:219-24.

3. Xue L, Wang L, Dong J, Yuan Y, Fan H, Zhang Y, et al. Risk factors of myasthenic crisis after thymectomy for thymoma patients with myasthenia gravis. Eur J Cardiothorac Surg. 2017;52:692-7.

4. Okereke IC, Kesler KA, Morad MH, Mi D, Rieger KM, Birdas TJ, et al. Prognostic indicators after surgery for thymoma. Ann Thorac Surg. 2010;89:1071-7.

5. Wolf AS, Flores RM. Extrapleural pneumonectomy for pleural malignancies. Thorac Surg Clin. 2014;24:471-5. 